\title{
When should we treat children with HIV?
}

\author{
James M. Oleske*
}

Romanelli et al. have reviewed the evolution of antiretroviral (ARV) therapy implemented between January 1998 and December 2001 at Universidade Federal de Minas Gerais (UFMG) and concluded that triple drug regimens were more effective than dual drug therapy. ${ }^{1}$ Their conclusion mirrors, in part, US pediatric guidelines for antiretroviral therapy in HIVinfected children. Initially developed in 1993, these guidelines have been updated periodically (last update: March 11, 2005) by the Working Group on Antiretroviral Therapy and Medical Management of HIV-Infected Children, composed of specialists caring for HIV-infected infants, children, and adolescents, convened by the National Resource Center at the François-Xavier Bagnoud Center, University of Medicine and Dentistry of New Jersey (UMDNJ); the Health Resources and Service Administration (HRSA); and the National Institutes of Health (NIH). ${ }^{2}$ These updated guidelines reflect the major strides being made in laboratory and clinical research including the introduction and development of new classes of drugs that allow for significant viral load reduction, leading to decreased disease progression and mortality. Although the pathogenesis of HIV infection and the general principles of therapy are the same for HIV-infected adults, adolescents, children and infants, antiretroviral treatment of HIV infection in pediatrics requires consideration of a number of factors specific to this population, including differences in drug pharmacokinetics and in virologic and immunologic markers compared with older patients, as well as age-related adherence issues. ${ }^{3}$

\footnotetext{
MD, MPH, Professor of Pediatrics, François-Xavier Bagnoud Center for Health and Human Rights, Boston, MA, USA. Director, Division of Allergy, Immunology \& Infectious Diseases, Department of Pediatrics, Medical School, University of Medicine and Dentistry of New Jersey (UMDNJ), Newark, NJ, USA.

Suggested citation: Oleske JM. When should we treat children with HIV? J Pediatr (Rio J). 2006;82:243-5.

doi:10.2223/JPED.1515
}

In infants who are found to be HIV-infected, the early institution of antiretroviral regimens has become the standard of care to reduce mortality and the development of resistance mutations while preserving immunologic function. 4,5 There are a number of issues that make treatment of HIV more problematic in the pediatric age group. These include the timing of HIV infection in infants, which occurs when their immune systems are developing and more susceptible to the negative consequences of HIV infection. The pharmacokinetics of the multiple drugs used in the treatment of HIV varies as infants mature through childhood and adolescence. This factor also accounts for more rapid disease progression in pediatric patients, and the requirement for lifelong treatment regimens during the course of normal childhood growth and development. Not only does the pharmacokinetics of antiretroviral drugs in pediatrics vary with age, it also appears that children suffer from enhanced acute as well as long-term drug toxicities. ${ }^{6}$ In addition, adherence issues in infants, children and adolescents can affect the effectiveness of antiretroviral regimens, mandating the development of drugs more specifically appropriate for children, including characteristics such as improved tolerability.

\section{Treatment recommendations Children younger than 12 months of age}

The risk of disease progression is inversely correlated with the age of the child, with the greatest risk of progression in the first year of life. By 12 months of age, about $50 \%$ of HIV-infected children develop moderate immune suppression, and $20 \%$ develop severe immune suppression. However, our ability to distinguish children at risk for rapid versus slower disease progression by clinical and laboratory parameters is very limited, especially among young children. ${ }^{7}$ Nonetheless, in general, infants who received early treatments with highly active antiretroviral therapy (HAART) were significantly less 
likely to progress to AIDS or death compared to those who received no therapy.

The Working Group recommends initiation of therapy for all infants under age 12 months who have clinical or immunologic symptoms of HIV disease, regardless of HIV RNA level, and that treatment be considered for selected infants who are asymptomatic and have normal immune parameters but have additional risk factors for possible rapid progression, such as high maternal viral load at delivery.

\section{Children 12 months of age and older}

The risk of rapid disease progression is lower in children over age 1 year. It is clear that children with clinical AIDS or severe immune suppression are at high risk of progression and death. However, children over age 12 months with mild to moderate clinical symptoms or moderate immune suppression are at lower risk for progression, and the levels of plasma HIV RNA provide useful information on the risk of disease progression. ${ }^{8}$

The Working Group recommends that treatment be started in children with AIDS or severe immune suppression and that treatment be considered in children with mild-tomoderate clinical symptoms, moderate immune suppression, or plasma HIV RNA level >100,000 copies/mL. Other factors which might affect treatment decision include safety, concern of adherence, rate of clinical, virologic, and immunologic progression.

\section{Choice of initial antiretroviral therapy}

Currently, there are 21 FDA-approved antiretroviral drugs for use in HIV-infected adults and adolescents, 13 of which have been approved for pediatric use. These drugs fall into four major classes: nucleoside or nucleotide analogue reverse transcriptase inhibitors (NRTIs, NtRTIs), non-nucleoside reverse transcriptase inhibitors (NNRTIs), protease inhibitors, and fusion inhibitors.

Recommendations regarding the optimal initial therapy for children are continually being modified as new data become available, new therapies or drug formulations are developed, and additional toxicities are recognized. The Working Group's recommendations on regimens for use in initial therapy were based on the following criteria: data on the durability of viral suppression and clinical and immunologic response; incidence and types of drug toxicity; availability and palatability of drug formulations for children; dosing frequency and associated food or fluid requirements; and the potential for drug interactions. The preferred regimens for initial therapy in children include the combinations of 2 NRTIs plus one of the preferred recommended protease inhibitors, or the combination of 2 NRTIs plus NNRTI. ${ }^{2}$

\section{Changing antiretroviral therapy}

The following are reasons to consider changing antiretroviral therapy:

1. Evidence of disease progression based on virologic, immunologic, or clinical parameters.

2. Toxicity or intolerance to the current regimen.

3. New data demonstrating that a different regimen is superior to the current regimen.

When treatment fails, one of the first things to consider is a possible adherence problem. In this case, directly observed therapy (DOT) may be necessary to distinguish between inadequate adherence and medication failure. Such adherence issues should be addressed before initiating a new regimen to increase the likelihood of success. In choosing a new antiretroviral regimen, the indications that warranted the change in therapy must first be considered (e.g., toxicity/intolerance vs. drug resistance vs. poor adherence). Unfortunately, data are limited regarding the pharmacokinetics, appropriate dosing, and safety of various combinations of antiretroviral drugs in children; extrapolation from clinical trial data obtained from adults becomes necessary in this case.

\section{Conclusion}

Management of HIV infection in the pediatric population is rapidly evolving and becoming increasingly complex. This is because the appropriate treatment of HIV-infected infants, children and adolescents requires consideration of a number of factors specific to these populations, including differences in drug pharmacokinetics and in virologic and immunologic markers compared with older patients, as well as age-related adherence issues.

Determination of the immunologic and virologic responses is essential for monitoring and modifying antiretroviral treatment in infected children and adolescent as well as adults. The choice of antiretroviral regimens should depend on the consideration of age-specific issues, efficacy of antiretroviral regimens extrapolated from clinical trials, as well as the consideration of factors associated with possible limitation of future treatment options, such as the presence of or potential future development of antiretroviral resistance.

\section{Personal observations}

The use of combination antiretroviral therapy in infants, children and adolescents has reduced morbidity and mortality, as well as improved quality of life of patients with perinatally acquired HIV, rendering HIV a chronic illness, with infected infants now surviving well into the third decade of their lives. ${ }^{6}$ 
With the introduction of protease inhibitors in 1996, mortality dropped by $70 \%$ per year in pediatric patients capable of tolerating chronic treatment with three drugs from at least two of the major antiretroviral classes. ${ }^{4}$ This achievement is attributable to the reduction in viral load and the consequent reconstitution of the immune system and drastic reduction in opportunistic infections that were previously frequently the causes of early death in perinatally HIV infected children. ${ }^{9}$ The restoration of the immune system has also enabled children to avoid treatment with antimicrobial drugs that have their own significant toxicities, and adds to the adverse drug profiles of primary antiretroviral agents.

Since the advent of the era of highly active antiretroviral therapy (HAART), and the implementation of treatment guidelines, we have experienced a remarkable change at the François-Xavier Bagnoud (FXB) Center for Prevention and Treatment of Perinatal HIV infection at the University of Medicine and Dentistry of New Jersey, Newark, New Jersey. In 1995, 35 of our patients died after childhoods marked by significant pain and suffering. ${ }^{10}$ In 2005, our program did not experience the death of a single patient despite an actual increase in the numbers of children and adolescents followed at the FXB Center. When our initial Children's Hospital AIDS Program was established in 1985, we had few expectations that any of the infants under our care would survive long enough to attend school. This year, one of our perinatally infected patients, now a young adult, graduated from medical school and is in training to become a pediatrician, specifically in infectious disease.

Now that HIV infection has become a chronic and not universally lethal illness, we need to redouble our efforts to maximize its prevention as well as its optimal treatment. This goal demands the availability and provision of prenatal care to every pregnant woman, so that her own disease can be well controlled, and both she and her future offspring can enjoy the benefits of proven therapeutic strategies to prevent perinatal transmission of HIV. This challenge is obviously made much more difficult because of the limited access to health care for underserved populations both in the United States and worldwide. Nonetheless, these barriers must be surmounted in order to avoid millions of infants being infected with this preventable disease. If we choose not to accept this challenge worldwide, death and ongoing suffering will negatively impact global stability. The primary approach to the prevention of perinatal infection worldwide will be the assured provision of ARV treatment/prophylaxis to pregnant HIV infected women; ${ }^{11}$ failing that, a therapeutic vaccine or other immune-based therapy that augments the benefits of antiretroviral treatment of the newborn will be required. Such immune-based therapies or therapeutic vaccines, when used in combination with more effective antiretroviral treatment may eradicate the last vestige of HIV, thus finally providing a cure for those who are now chronically infected.

Although some information regarding the efficacy of antiretroviral drugs for children can be extrapolated from clinical trials involving adults, concurrent pediatric clinical trials are needed to determine the impact of drugs on specific manifestations of HIV infection in children, including growth, development and potential unique long-term complications. Management of HIV infection in pediatric populations is rapidly evolving and becoming increasingly complex. Imperfect efficacy, significant toxicities, and ever emerging resistance make the development of new and better antiretroviral agents necessary. Pharmaceutical companies and the federal government must collaborate to ensure that drug formulations suitable for administration to infants and children become available at the same time that new drugs are evaluated and approved for use by adults.

\section{References}

1. Romanelli RM, Pinto JA, Melo LJ, Vasconcelos MA, Pereira RM. Effectiveness of dual and triple antiretroviral therapy in the treatment of HIV-infected children. J Pediatr (Rio J). 2006;82: 260-5.

2. Working Group on Antiretroviral Therapy: National Pediatric HIV Resource Center. Antiretroviral therapy and medical management of the human immunodeficiency virus-infected child. Pediatr Infect Dis. 1993;12:513-22.

3. Gray L, Newell ML, Thorne C, Peckham C, Levy J. Fluctuations in symptoms in human immunodeficiency virus-infected children: the first 10 years of life. Pediatrics. 2001;108:116-22.

4. Gortmaker SL, Hughes M, Cervia J, Brady M, Johnson GM, Seage GR 3rd, et al. Effect of combination therapy including protease inhibitors on mortality among children and adolescents infected with HIV-1. N Engl J Med. 2001;345:1522-8.

5. Soh $\mathrm{CH}$, Oleske JM, Brady MT, Spector SA, Borkowsky W, Burchett SK, et al. Long-term effects of protease-inhibitorsbased combination therapy on CD4 T-cell recovery in HIV-1infected children and adolescents. Lancet. 2003;362:2045-51.

6. Brogly S, Williams P, Seage GR 3rd, Oleske JM, Van Dyke R, McIntosh K, et al. Antiretroviral treatment in pediatric HIV in the United States: from clinical trials to clinical practice. JAMA. 2005;293:2213-20.

7. Abrams EJ, Wiener J, Carter R, Kuhn L, Palumbo P, Nesheim S, et al. Maternal health factors and early pediatric antiretroviral therapy influence the rate of perinatal HIV-1 disease progression in children. AIDS. 2003;17:867-77.

8. Galli L, DeMartino M, Tovo PA, Gabiano C, Zappa M. Predictive value of the HIV paediatric classification system for the longterm course of perinatally infected children. Int J Epidemiol. $2000 ; 29: 573-8$.

9. Gona P, Van Dyke RB, Williams PL, Dankner WM, Chernoff MM, Nachman SA, et al. Incidence of opportunistic and other infections in HIV-infected children in the HAART era. JAMA. 2006;296:292300.

10. Gaughan DM, Hughes MD, Seage GR, Selwyn P, Carey VJ, Gortmaker SL, et al. The prevalence of pain in pediatric HIV/ AIDS as reported by participants in the Pediatric Late Outcomes Study (PACTG 219). Pediatrics. 2002;109:1144-52.

11. Connor EM, Sperling RS, Gelber R, Kiselev P, Scott G, O'Sullivan $M J$, et al. Reduction of maternal-infant transmission of human immunodeficiency virus type 1 with zidovudine treatment. Pediatric AIDS Clinical Trials Group Protocol 076 Study Group. N Engl J Med. 1994;331:1173-80. 